\section{JOHN VON NEUMANN}

John von Neumann

Collected Works. General Editor: Prof. A. H. Taub. Vol. 4: Continuous Geometry and other Topies. Pp. $x+516$. Vol. 5: Design of Computers, Theory of Automata and Numerical Analysis. Pp. viii +785 . Vol. 6: Theory of Games, Astrophysics, Hydrodynamics and Meteorology. Pp. viii +538. (London and New York: Pergamon Press, 1962 and 1963.) 100s. net each volume.

A SKETCH of the life of John von Neumann and a review of Volumes 1 and 2 of his Collected Works appeared in Nature, 196, 100 (1962). Volumes 4, 5 and 6 were published during 1962-63 (reference should again be made to the authoritative commentaries on von Neumann's life and work mentioned in the aforementioned review).

The Collected Works include all von Neumann's published papers and also contain a bibliography and reviews of a number of his manuscripts, such as lecture notes not suitable for publication, and now in the library of the Institute for Advanced Study, Princeton.

Volume 4 contains a collection of papers of widely varying scope, some having lasting importance and a few having perhaps only minor permanent significance. The group of papers on continuous geometries and geometries without points are important and lasting contributions to pure mathematics. Von Neumann's interest in such geometries was at least partly due to his repeated efforts to improve the mathematical formulation of the foundations of quantum mechanics and to his hope that these geometries might give a route. However, this hope was not realized. Two other fields in which there are important papers are continuations of von Neumann's work on topological vector spaces (linear transformations in Banach spaces) and a new proof of the existence and uniqueness of Haar measure on compact groups. One other extremely important paper is the solution of Hilbert's fifth problem for compact groups. The five papers at the end of the volume contain answers worked out by von Neumann to certain ballistics, statistical and aiming problems put to him in 1940.

Volume 5 is concerned with von Neumann's contributions to the logic, specification and application of large computers. In one sense, the papers are of ephemeral technical interest because the logic of automata is now so widely studied that early work, however basic and farsighted, must inevitably be submerged in world-wide progress. However, the contents of Volume 5 show how vital were the contributions made by von Neumann in making the computer the bulldozer of applied mathematics. The paper "On the Principles of Large Scale Computing Machines" was never published but the contents formed the substance of a lecture delivered many times by von Neumann. His spoken words had a great impact and persuaded many groups and laboratories with specialized interests, ranging among atomic energy, ballistics and aerodynamics, space research, long-range weather forecasts and high-energy physics (to mention only some examples) that a large computer was a powerful new tool which could be usod flexibly and rapidly to great advantage.

The moro deeply mathematical papers in this volume are those on the solution of linear systems of high order, on the inversion of matrices of high order, and on the probability logic of synthesizing reliable 'organisms' from unreliable components. There are also several interesting discussions of practical problems where tho methods of numerical solution of the basic equations are discussed, and where numerical answers to specific problems were obtained by using a computer. One example is the twodimensional motion from rest of one incompressible fluid resting on another eight times less dense, both fluids contained in a box and the interface initially plane and sloping. The infinitesimal motion has Taylor instability, but the object of the calculation was to study the finite motion of gross instability. Other examples are a neutron diffusion problem and the computation of the values of $e$ and $\pi$ to 2,000 decimal places and the statistical randomness of the digits.

Volume 6 contains nine papers on the theory of games. These papers are woven into the text of the book with Morgenstern on games and economic behaviour. The theory has had a wide influence; and, parenthetically, it is interesting to note, for example, that von Neumann's mathematical ideas about games theory have led to a computer service which is now available in the United States to optimize the distribution of investments in a portfolio, the customer stating his estimates of the probability of swings either way in the market value of each share.

The remaining four-fifths of Volume 6 give von Neumann's mathematical work on various problems of fluid mechanics, usually problems connected with shock waves and blast. Von Neumann was one of a number of brilliant academic scientists asked to help the United States war effort through flexible consultative links with several of the United States agencies (the Office of Scientific Research and Development, the Bureau of Ordnance of the Navy Department, the Manhattan District, etc.). He took to the theory of compressible fluids like a duck to water. His theory of the explosive detonation front is well known. He developed the similarity theory of the instantaneous point source explosion in a perfect gas, a theory given independently by G. I. Taylor. However, von Neumann also gave a numerical method of continuing the solution beyond the stage where the 'strong shock' relations are losing their accuracy.

Another of von Neumann's ideas is well known-the artifice of solving certain types of shock-wave problems numerically on a computer, step by step in time, without the operator having to adjust the conditions at the shock front at each step. Von Neumann and Richtmyer merely put in a viscosity type term, with a fictitious value so chosen for the viscosity that the shock front was continuous, although with rapidly varying variables, spread over a few mesh points. Volume 6 also contains a series of interesting papers on the oblique reflexion of shocks. A plane shock wave is not reflected acoustically by a rigid plane surface: sometimes the plane-reflected shock and the incident shock meet at the surface, and sometimes a Mach $Y$ forms. (Mach was the first to notice the phenomenon experimentally in 1878.) These idealized mathematical considerations on shock-wave reflexions have a bearing on the mechanies of a system in which an explosive charge is detonated in air above the ground; and von Neumann discusses this system. The volume concludes with summarios of certain reports, lectures and statements made by von Neumann on a range of topies, such as the role of science in society, the chance of man surviving technology and other political or sociological problems.

W. G. PENNEY

\section{TYCHO BRAHE}

\section{Tycho Brahe}

A Picture of Scientific Life and Work in the Sixteenth Century. By Dr. J. L. E. Dreyer. Pp. xvi +405 . (New York: Dover Publications, Inc.; London: Constable and Co., Ltd. Dover Edition, 1963.) 16s.; 2.0 dollars.

TYCHO BRAHE is a reprint of a book first published in 1890. The name of J. L. E. Dreyer of the Armagh Observatory is not well remembered to-day, but he was one of the most scholarly writers on astronomy and its history at the end of the nineteenth century and later; and it is remarkable how little has been added to our knowledge of Tycho Brahe since the publication of this 